\title{
Estimating groundwater vulnerability to pollution using a modified DRASTIC model in the Kerman agricultural area, Iran
}

\begin{abstract}
Groundwater contamination from intensive fertilizer application affects conservation areas in a plain. The DRASTIC model can be applied in the evaluation of groundwater vulnerability to such pollution. The main purpose of using the DRASTIC model is to map groundwater susceptibility to pollution in different areas. However, this method has been used in various areas without modification, thereby disregarding the effects of pollution types and their characteristics. Thus, this technique must be standardized and be approved for applications in aquifers and particular types of pollution. In this study, the potential for the more accurate assessment of vulnerability to pollution is achieved by correcting the rates of the DRASTIC parameters. The new rates were calculated by identifying the relationships among the parameters with respect to the nitrate concentration in groundwater. The methodology was implemented in the Kerman plain in the southeastern region of Iran. The nitrate concentration in water from underground wells was tested and analyzed in 27 different locations. The measured nitrate concentrations were used to associate and correlate the pollution in the aquifer to the DRASTIC index. The Wilcoxon rank-sum nonparametric statistical test was applied to determine the relationship between the index and the measured pollution in Kerman plain. Also, the weights of the DRASTIC parameters were modified through the sensitivity analysis. Subsequently, the rates and weights were computed. The results of the study revealed that the modified DRASTIC model performs more efficiently than the traditional method for nonpoint source pollution, particularly in agricultural areas. The regression coefficients showed that the relationship between the vulnerability index and the nitrate concentration was $82 \%$ after modification and $44 \%$ before modification. This comparison indicated that the results of the modified DRASTIC of this region are better than those of the original method.
\end{abstract}

Keyword: GIS; Groundwater; Hydrogeology; Kerman plain; Modified DRASTIC; Sensitivity analysis; Vulnerability 